\title{
A streamlined mass spectrometry-based proteomics workflow for large scale FFPE tissue analysis
}

Coscia, Fabian; Doll, Sophia; Bech, Jacob Mathias; Schweizer, Lisa; Mund, Andreas;

Lengyel, Ernst; Lindebjerg, Jan; Madsen, Gunvor Iben; Moreira, José M A; Mann, Matthias

Published in:

Journal of Pathology

DOI:

$10.1002 /$ path.5420

Publication date:

2020

Document version

Publisher's PDF, also known as Version of record

Document license:

CC BY

Citation for published version (APA):

Coscia, F., Doll, S., Bech, J. M., Schweizer, L., Mund, A., Lengyel, E., Lindebjerg, J., Madsen, G. I., Moreira, J. M. A., \& Mann, M. (2020). A streamlined mass spectrometry-based proteomics workflow for large scale FFPE tissue analysis. Journal of Pathology, 251, 110-112. https://doi.org/10.1002/path.5420 


\title{
A streamlined mass spectrometry-based proteomics workflow for large-scale FFPE tissue analysis
}

\author{
Fabian Coscia 1,2†미, Sophia Doll ${ }^{2+,}$, Jacob Mathias Bech ${ }^{3}$, Lisa Schweizer ${ }^{2}$, Andreas Mund ${ }^{2}$, Ernst Lengyel ${ }^{4}$, \\ Jan Lindebjerg ${ }^{5}$, Gunvor Iben Madsen ${ }^{6}$, José MA Moreira ${ }^{3}$ and Matthias Mann ${ }^{1,2 *}$ \\ I Clinical Proteomics Group, The Novo Nordisk Foundation Center for Protein Research, Faculty of Health and Medical Sciences, University of \\ Copenhagen, Copenhagen, Denmark \\ 2 Department of Proteomics and Signal Transduction, Max Planck Institute of Biochemistry, Martinsried, Germany \\ 3 Section for Molecular Disease Biology, Department of Drug Design and Pharmacology, Faculty of Health and Medical Sciences, University of \\ Copenhagen, Copenhagen, Denmark \\ ${ }^{4}$ Department of Obstetrics and Gynecology, Section of Gynecologic Oncology, University of Chicago, Chicago, IL, USA \\ 5 Lillebaelt Hospital, Vejle Hospital, Department of Pathology, Vejle, Denmark \\ ${ }^{6}$ Department of Pathology, Odense University Hospital, Odense, Denmark
}

*Correspondence to: M Mann, Max Planck Institute of Biochemistry, D-82 52 Martinsried, Germany. E-mail: mmann@biochem.mpg.de

TThese authors contributed equally.

New address: OmicEra Diagnostics GmbH, Am Klopferspitz 19, D-82I52 Planegg, Germany.

\begin{abstract}
Formalin fixation and paraffin-embedding (FFPE) is the most common method to preserve human tissue for clinical diagnosis, and FFPE archives represent an invaluable resource for biomedical research. Proteins in FFPE material are stable over decades but their efficient extraction and streamlined analysis by mass spectrometry (MS)-based proteomics has so far proven challenging. Herein we describe a MS-based proteomic workflow for quantitative profiling of large FFPE tissue cohorts directly from histopathology glass slides. We demonstrate broad applicability of the workflow to clinical pathology specimens and variable sample amounts, including low-input cancer tissue isolated by laser microdissection. Using state-of-the-art data dependent acquisition (DDA) and data independent acquisition (DIA) MS workflows, we consistently quantify a large part of the proteome in $100 \mathrm{~min}$ single-run analyses. In an adenoma cohort comprising more than 100 samples, total workup took less than a day. We observed a moderate trend towards lower protein identification in long-term stored samples ( $>15$ years), but clustering into distinct proteomic subtypes was independent of archival time. Our results underscore the great promise of FFPE tissues for patient phenotyping using unbiased proteomics and they prove the feasibility of analyzing large tissue cohorts in a robust, timely, and streamlined manner.

(c) 2020 The Authors. The Journal of Pathology published by John Wiley \& Sons Ltd on behalf of Pathological Society of Great Britain and Ireland.
\end{abstract}

Keywords: biobank samples; proteomics; mass spectrometry; FFPE tissue; high-throughput, protocol, biomarker discovery; adenoma

Received 23 September 2019; Revised 2 March 2020; Accepted 4 March 2020

No conflicts of interest were declared.

\section{Introduction}

Hospitals routinely archive biopsied tissue specimens that are collected for diagnostic purposes through formalin fixation and paraffin-embedding (FFPE). FFPE is an economical archival choice, as tissues can be stored at great density at room temperature (RT) for years or decades while maintaining integrity for pathology analyses [1]. An estimated 500 million FFPE cancer tissues are stored to date, and this number is rapidly rising [2]; these present an invaluable resource for studying molecular mechanisms underlying diseases and testing potential biomarkers and discovering new ones. The long- term storage of FFPE specimens implies that they are often associated with a plethora of clinical data, including histology reports, treatment, and patient outcomes. Such metadata are of particular importance for integration of retrospective clinical and molecular data to improve patient phenotyping and stratification. FFPE sample preparation involves tissue fixation and embedding in an inert matrix, which makes oligonucleotidebased analysis difficult and often impossible [3,4]. Antibody-based histological methods are routinely performed but rely on the preservation of the relevant epitopes, which can also be very challenging and influence quantitation [5]. Because of these difficulties and 
the fact that mass spectrometry (MS)-based proteomics demands clean peptide samples to be introduced to the mass spectrometer, it came as a surprise when we and others showed that FFPE samples are indeed very well suited to this technology, including the analysis of post-translational modifications (PTMs) [6,7]. Furthermore, carcinomas could be analyzed at great complexity, in excess of 10000 expressed proteins [8]. These and other studies together established that FFPE tissues show similar qualitative and quantitative proteomic properties as fresh frozen ( $\mathrm{FrFr}$ ) tissues, further highlighting the potential of FFPE tissue analysis for biomedical research $[6,9,10]$.

Recent technological progress of MS-based proteomic methods now enables the unbiased and large-scale investigation of proteomes at ever greater depth [11]. As a result, in-depth proteomic analysis of FFPE tissues in a clinical context has now become feasible. Using MS-based proteomics on archived FFPE tissues, we recently profiled more than 9000 protein groups from metastatic ovarian cancer (OvCa) FFPE tissues and identified a novel prognostic disease biomarker, 'CT45', causally linked to patient long-term survival [12]. Using biobanked frozen tissue samples, studies from the Clinical Proteomic Tumor Analysis Consortium (CPTAC) highlighted the importance of integrating proteomic and phosphoproteomic data with genomic information to uncover functional consequences of genomic mutations and identify proteogenomic subtypes linked to patient outcome [13,14]. Despite these promising MS technology developments and improved protein extraction protocols from FFPE tissues, streamlined workflows for efficient processing of hundreds of tissue samples in a highly parallelized and robust manner are not yet available. Hence, most proteomic studies including our own have so far focused on relatively small patient cohorts (fewer than 60 samples) and required large amounts of biological starting materials, often serial tissue sections (5- to $10-\mu \mathrm{m}$ thick). Typically, strong detergents such as sodium dodecyl sulfate (SDS) are used in lysis buffers, combined with boiling times up to a few hours, to efficiently reverse formalin crosslinks and extract and solubilize proteins [15]. These methods, however, come with some caveats as detergents are incompatible with LC-MS/MS downstream analysis and therefore require detergent removal steps that can cause sample loss and also lower reproducibility. Therefore, most detergent-based protocols are incompatible with ultra-low sample input, such as laser-capture microdissected (LCM) samples, which often range from only a few hundred to a few thousand cells. These sub-microgram protein amounts are easily lost in detergent clean-up steps and highlight the need for alternative protocols optimized for low sample amounts. Thus, a workflow enabling the processing of both low-input and high-input samples would be highly desirable. This is further illustrated from a pathologist's perspective; depending on disease stage, anatomic location, and pharmacologic intervention or due to surgical circumstances, tissue availability can be highly variable and often limited. Although pre-cancerous lesions can comprised only hundreds of cells, primary and metastatic tumors are typically available in larger quantities. Workflows that are not biased towards either end would therefore be of great clinical value for the investigating of disease mechanisms at the global proteome level.

Herein we report the development of a robust, scalable, and high-throughput workflow for quantitative proteomic profiling of patient-derived FFPE tissue samples. Consistent with previous observations [16,17], we show that detergent-free protocols are excellent for proteomic profiling of FFPE tissues in a time- and costeffective manner. Sample preparation is carried out with MS-compatible buffers to facilitate tissue lysis, protein extraction, and tryptic digestion in the same tube [18-20]. We developed this approach into a generic strategy applicable to large clinical sample cohorts and provide a detailed 'how-to' guide, from sample collection and preparation to MS measurement. We successfully applied our workflow to an adenoma cohort of more than 100 samples, thereby validating its suitability for large sample cohorts and its ability to reveal new insights into disease biology.

\section{Materials and methods}

\section{Sample collection}

All samples were human FFPE tissues collected with informed consent (University of Chicago Institutional Review Board-approved protocol [13372] for ovarian cancer (OvCa), Charité University Hospital Berlin for glioma [EA2/101/08], University Hospitals Jena for acute myeloid leukemia (AML) [Vote 3477-3406/12], University Medical Centre Mannheim for urachal carcinoma [2015-540-MA], Health Research Ethics Committee of the Capital Region of Denmark for colorectal adenomas [H-16022392]), and in accordance with the Declaration of Helsinki.

\section{Sample preparation for MS analysis}

A detailed sample collection and preparation protocol for FFPE tissues is provided in the supplementary material, Supplementary materials and methods. For AML cell collection, newly diagnosed untreated de novo AML patient cells were obtained from peripheral blood samples. Samples were enriched for mononuclear cells using density-gradient centrifugation for $30 \mathrm{~min}$ at $400 \times \mathrm{g}$ using BIOCOLL separating solution (Density 1.077, Biochrom, Berlin, Germany). The blast-enriched peripheral blood mononuclear cells (PBMCs) were washed in $1 \mathrm{X}$ phosphate-buffered saline once and cryopreserved at $-150^{\circ} \mathrm{C}$.

\section{Liquid chromatography-mass spectrometry (LC-MS/MS) analysis}

Nanoflow LC-MS/MS analysis of tryptic peptides was conducted on a quadrupole Orbitrap mass spectrometer 
(Q Exactive HF-X, Thermo Fisher Scientific, Bremen, Germany) [21] coupled to an EASY nLC 1200 ultrahigh-pressure system (Thermo Fisher Scientific) via a nano-electrospray ion source. About $300 \mathrm{ng}$ of peptides were loaded onto a $50 \mathrm{~cm}$ high performance liquid chromatography (HPLC)-column (75 $\mu \mathrm{m}$ inner diameter, New Objective, Woburn, MA, USA; in-house packed using ReproSil-Pur C18-AQ 1.9- $\mu$ m silica beads; Dr Maisch GmbH, Ammerbuch, Germany) and measured over a total gradient length of 100 min with increasing buffer B ( $80 \%$ acetonitrile $[\mathrm{ACN}]$ and $0.1 \%$ formic acid; Merck, Darmstadt, Germany) concentration. The mass spectrometer was operated in data dependent acquisition (DDA) and data independent acquisition (DIA) mode, as specified in the supplementary material.

\section{MS data analysis}

In brief, DIA raw files were analyzed with Spectronaut Pulsar X software (Biognosys, Schlieren, Switzerland, version 12.0.20491.17) under default settings for targeted DIA analysis with 'mutated' as decoy method. DDA raw files were processed in the MaxQuant environment [22] (version 1.5.0.38 and 1.6.7.0). The human UniProtKB database (October 2017 and 2019, UP000005640_9606) was used as the forward database and the automatically generated reverse database for the decoy search.

\section{Statistical analysis}

All statistical and bioinformatic analyses were performed using Perseus [23] or the $\mathrm{R}$ framework (https://www.r-project.org/). Missing values (those displayed as 0 or ' $\mathrm{NaN}$ ' in the MaxQuant output) were imputed based on a normal distribution (width $=0.3$; downshift $=1.8$ ). To calculate the percentage of missing values per data matrix, the number of valid values (those not displayed as 0 or ' $\mathrm{NaN}$ ' in the MaxQuant output) was divided by the total number of possible values (samples $\mathrm{x}$ proteins) for a given matrix. Consensus clustering was performed based on the ConsensusClusterPlus R library [24]. The 1000 most variably expressed protein groups (calculated by median absolute deviation) were used for consensus clustering. The number of clusters, $\mathrm{k}$, was varied from 2 to 8 with 1000 resamplings. Hierarchical clustering was used based on Pearson correlations as a distance metric. The Consensus Cumulative Distribution Function (CDF) plot and Delta Area plot were used to assess the optimal number of clusters.

\section{Immunohistochemistry}

Immunohistochemistry was carried out as described previously [25]. All antibodies were validated with negative and positive control tissue as described in supplementary material, Supplementary materials and methods.

\section{Results}

Proteomic sample preparation of archived biobank tissue in 96-well format

To be useful for large and diverse FFPE tissue cohorts, we optimized our recently described workflow for laser-capture microdissected (LCM) FFPE samples [26] to a 96-well format (Figure 1A). We further compared our workflow to two widely applied protocols for FFPE tissue proteomics [9,27] (FASP and RapiGest-based). We worked with macrodissected and microdissected FFPE sections on glass slides to test the applicability to varying FFPE tissue amounts. We here refer to 'macrodissections' as razor-blade scraped areas from FFPE sections, whereas by 'microdissection' we mean LCM tissue from glass membrane slides (Figure 1B, supplementary material, Figure S1A). We first macrodissected similar tumor areas $(\sim 5 \times 5 \mathrm{~mm})$ by scraping from two consecutive $10 \mu \mathrm{m}$ thick sections of the same high-grade serous ovarian cancer (OvCa) specimen. We then processed the collected tissues with our organic solvent (2, 2, 2-trifluoroethanol; TFE) or previously described SDS-based workflow [12] for relative comparison of the two methods. We noticed that long heating times $(90 \mathrm{~min})$ in the SDS buffer fully resolved FFPE tissue in contrast to the TFE-based lysis. After overnight tryptic digestion, however, the tissue was fully resolved in both conditions, with no noticeable undigested material. Following peptide clean-up, we found a 2.3-fold higher peptide yield using the TFEbased protocol (supplementary material, Figure S1B), indicating both improved protein and peptide recovery $[16,28]$ and less protein loss during detergent clean-up steps. We injected $0.3 \mu \mathrm{g}$ of each peptide sample on a quadrupole Orbitrap mass spectrometer (Q Exactive HF-X) and analyzed them in 100 min single-run DDA mode (top15). Of the 5041 identified protein groups, 92\% were found with both protocols, indicating that protein extraction was highly comparable despite the noticeable differences in tissue dissolving directly after heating. The average proteome correlation was 0.95 (Pearson $\mathrm{r}$ ) based on label-free quantification (MaxLFQ) values [29] (supplementary material, Figure S1C), showing high proteome similarity. For the protocol comparison (FASP and RapiGest based), we macrodissected three identical areas from three consecutive $10 \mu \mathrm{m}$ sections of a glioma sample and processed them in parallel (Figure 1C, Figure S1D). LC-MS/MS analysis showed uniform total ion chromatograms for all three protocols (Figure 1D), resulting in high proteome correlations (Pearson $r=0.97-0.98$, Figure $1 F$ ) with $>90 \%$ of the proteins quantified with all three methods (Table 1). Notably, the TFE-based protocol resulted in the highest average and total number of peptide and protein identifications while also being the most time- and cost-effective of the three methods (Figure 1C, E and Table 1). To assess differences in protein extraction across protocols, we calculated the proportion of quantified proteins annotated to originate from different cellular compartments. This 
revealed almost identical cellular compartment proportions for the different preparations of the same OvCa or glioma specimen $( \pm 0.4 \%)$, and only minor differences (1-4\%) compared to fresh frozen tissue (supplementary material, Figure S1E). Likewise, we did not observe any differences in the abundance of DNA and chromatin bound proteins, which represent a particular challenge for formaldehyde-fixed samples due to stable DNA-protein crosslinks that may hinder peptide identification (supplementary material, Figure S1F). Together, these data demonstrate efficient protein extraction using our organic solvent-based workflow, including chromatin-bound proteins.

Formaldehyde crosslinking of FFPE tissue preserves tissue architecture, predominantly via stable methylene bridges between basic amino acid residues [30,31]. High proteome coverage of FFPE tissue therefore requires efficient formaldehyde de-crosslinking to reverse unwanted and variable chemical modifications that may obscure peptide identification. We used pFind, an 'open modification search' algorithm [32], to screen for the most abundant protein modifications present in the analyzed FFPE tissues. In line with previous reports [17,33], methionine oxidation and lysine methylation were the most abundant variable modifications compared to FrFr tissue (supplementary material, Figure S1G). Lysine methylation is a frequent protein modification in FFPE tissue, accounting for around 2-6\% of all identified peptides [33]. To ensure most efficient de-crosslinking, we opted for long heating times (90 $\mathrm{min}$ ) in combination with ultrasound homogenization (supplementary material, Figure S2A) and high Tris concentrations (300 $\mathrm{mM})$ in the lysis buffer [34]. Independent of the protocol (FASP, RapiGest or TFE), 4-6\% of all peptides were lysine methylated, indicating similar de-crosslinking efficiencies. Among the lysine methylation sites that were identified as methylated and unmethylated, the methylated state was in median $70 \%$ less abundant than the unmodified state in the OvCa tissue and $90 \%$ less for glioma, respectively (supplementary material, Figure $\mathrm{S} 1 \mathrm{H}$ ), indicating that differences in lysine methylation across samples should only marginally affect global proteome quantification.

Despite its beneficial chemical properties for improved protein and peptide extraction [16,28], TFE is a hazardous substance posing a higher safety risk compared to most other protein extraction methods. ACN is an alternative organic solvent, which shares similar physicochemical properties while being less hazardous. Replacing TFE with ACN in our FFPE workflow resulted in similar peptide and protein yield, further reflected by high proteome correlations (Pearson $r=0.97$ ) (supplementary material, Figure S1I-K). Taken together, these results demonstrate that our sample-processing protocol enables robust proteomic profiling of FFPE tissues based on a streamlined 'single-tube' workflow.

\section{FFPE tissue workflow is broadly applicable to various tumor types}

We next applied our workflow to FFPE sections from different tissue types (OvCa, glioma, colorectal adenoma, and urachal carcinoma). We chose these four tissue types because they strongly vary in their origin and prevalence while showing different grades of stroma content. This allowed us to test the broad applicability of our workflow to various tumor types. Before collection, all tissue sections were $H \& E$ stained and subjected to microscopic inspection to identify neoplastic regions of interest that were subsequently macrodissected or subjected to LCM (Figure 1B and supplementary material, Figure S1A). Excised tissue pieces were transferred into polymerase chain reaction (PCR) tubes, allowing highly parallelized 'single-tube' sample preparation in a 96-well format (Figure 1A, B, supplementary material, Figure S2A). MS analysis revealed excellent chromatographic signal for micro- and macrodissected samples with comparable total ion currents (TICs), independent of cancer origin or collection type (supplementary material, Figure S2B). We identified a similar number of peptides (on average 37 321) and protein groups (on average 4933) for micro- and macrodissected samples in 100 min DDA single-run analysis, demonstrating the broad applicability of our workflow (Figure 2A). To assess quantitative reproducibility, we compared the proteomes of five LCM FFPE tissues obtained from two advanced-stage high-grade serous OvCa (HGSOC) patients. We hypothesized that proteomic differences across patients would be larger than differences across anatomic sites from the same patient. Indeed, Pearson correlation coefficients were high for three biological replicates of the same patient (0.94-0.97), including primary (ovarian, OV) and metastatic (omental, OM) $\mathrm{OvCa}$, and somewhat lower between patients $(0.86-$ 0.88) (supplementary material, Figure S2D). This result is consistent with our recent data demonstrating that the HGSOC tumor proteome is driven by patientspecific protein signatures independent of anatomic site [26]. Principal component analysis (PCA) of glioma, colorectal adenoma, $\mathrm{OvCa}$, and urachal carcinoma clearly grouped them according to their tissue of origin in the first and second components (Figure 2B and supplementary material, Figure S2C). The segregation was driven by known marker proteins such as the epithelial OvCa markers PAX8, MSLN, FOLR1, and MUC1. Brain-specific proteins like MBP, PLP1, and SLC1A2 were most abundant in glioma, and the adenoma/urachus group showed high expression of the proteins AGR2, LGALS4, S100P, and CDH17, which are known intestinal cell-signaling and adhesion proteins (supplementary material, Figure S2C).

Quantitative completeness is an important aspect of proteomics and represents a particular analytical challenge in DDA strategies due to partially stochastic peptide sequencing. This is particularly true for single-run approaches that aim to quantify a large proportion of the cellular proteome without additional peptide prefractionation (which may not be appropriate for very low-input sample amounts or for reasons of limited total measurement time). To tackle this challenge, we evaluated different MS acquisition modes that could be combined with our workflow and increase our overall 
A

Biobank samples Sample processing

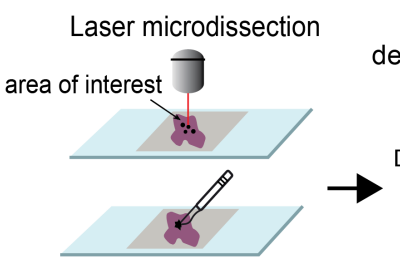

Macroscopic dissection

B

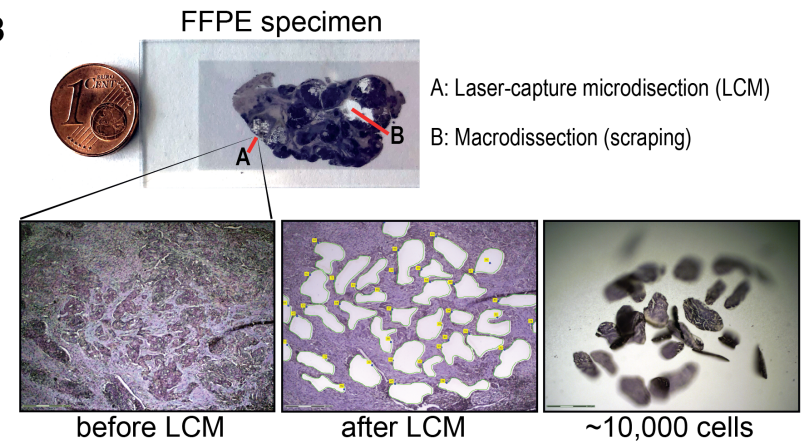

D

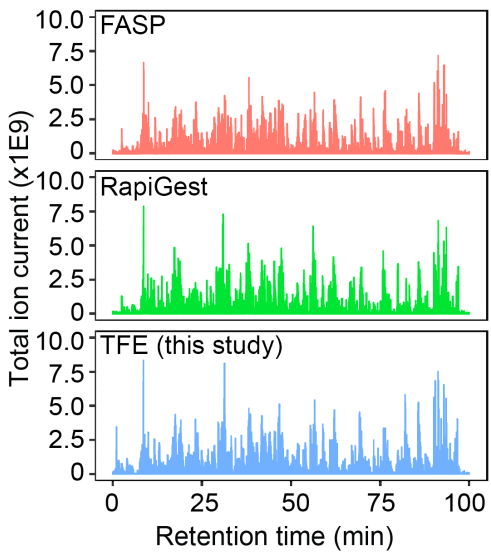

E

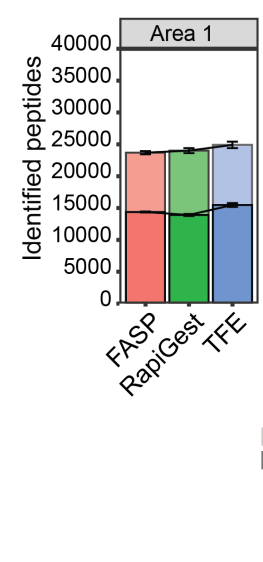

LC MS-MS

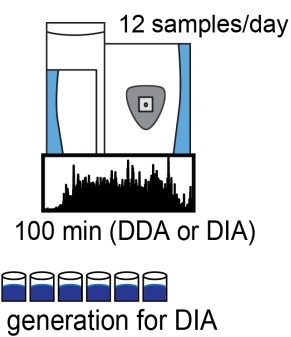

Library generation for DIA

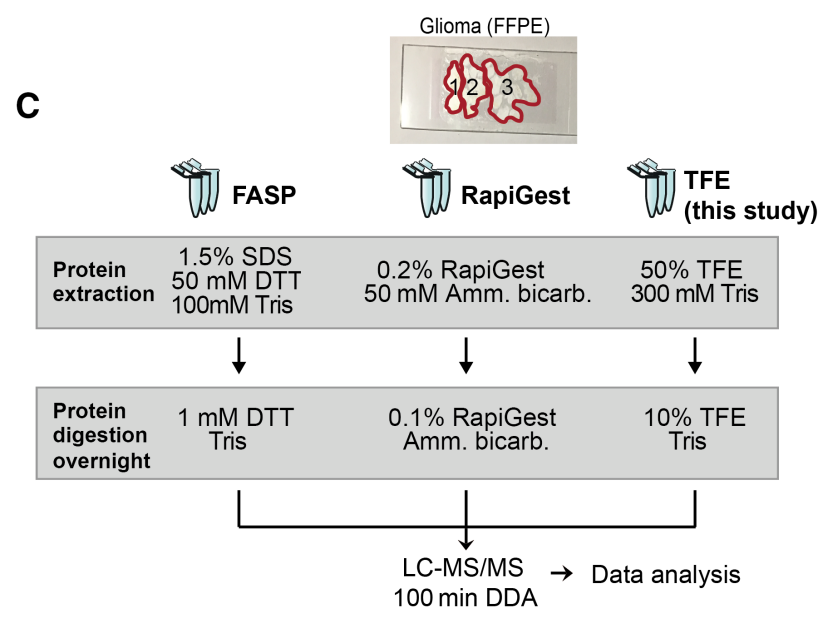

F

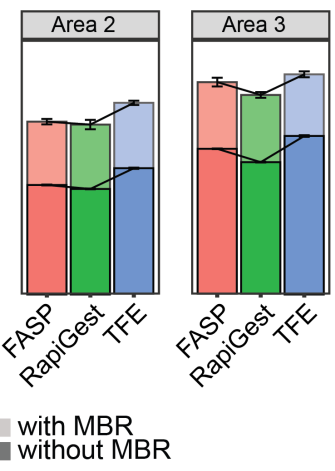

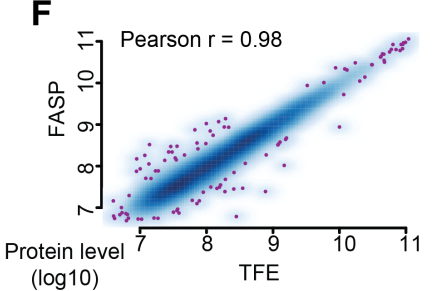

$(\log 10)$

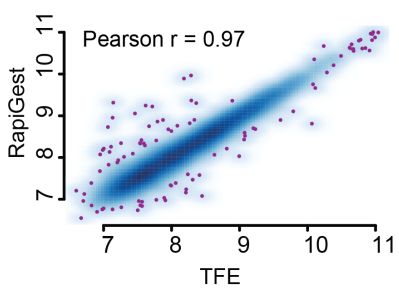

Figure 1. Overview of the mass spectrometry-based workflow for FFPE tissue analysis in 96-well format. (A) Streamlined FFPE workflow. FFPE tissues are collected from glass slides either by scraping or laser-capture microdissection (LCM), followed by 'single-tube' sample processing and MS-based proteomic analysis. (B) FFPE tissue collection techniques. Minute sample amount can be either collected by (A) LCM or (B) scraping from a histopathology glass slide. Images before and after LCM are shown in the first two lower panels. The third lower panel shows the collected LCM tissue regions, corresponding to an estimated number of 10000 cancer cells, calculated based on the total volume of dissected tissue. (C) Upper panel: Glioma FFPE tissue used for protocol comparison (FASP, RapiGest, TFE [this study]). For each protocol, three areas (labeled 1-3) from three consecutive $10 \mu \mathrm{m}$ sections of the same tissue were collected by macrodissection, processed, and analyzed by LC-MS/MS. Lower panel: Overview of protocols (FASP, RapiGest, TFE [this study]) used for comparison. (D) Total ion chromatogram of glioma tissue prepared with the three protocols. (E) Number of identified peptides reported by MaxQuant for 100 min single-run measurements. Each tumor area was measured as injection triplicates and mean values for each method were plotted with standard deviations as error bars. Identifications are shown with and without the 'match-between-runs' (MBR) feature of MaxQuant. (F) Quantitative comparison of glioma proteomes obtained by the FASP, RapiGest or TFE (this study) method. R correlations are Pearson correlation values.

identification rates. We first used the recently described 'BoxCar' scan mode, which allocates dynamic MS1 injection times based on precursor abundance [35]. Using BoxCar acquisition, the number of quantified protein groups increased on average by $7 \%$ in 100 min singleshot analysis resulting in 5419 and 5257 protein groups quantified in adenoma and glioma tissues, respectively (supplementary material, Figure S2E).
With the recent increased scan speed of Orbitrap analyzers, this platform has become very attractive for DIA strategies [36]. In DIA, the entire sample complexity is in principle captured by cycling of the quadrupole selection window over the entire $\mathrm{m} / \mathrm{z}$ range in pre-defined segments, thereby recording MS2 information irrespective of precursor intensity. Although proteome coverage was previously a major bottleneck for DIA single-run 
Table 1. Proteomic results for glioma tissue prepared with three different sample preparation protocols (related to Figure 1)

\begin{tabular}{|c|c|c|c|c|c|c|}
\hline & & \multirow[b]{2}{*}{ Recovered peptide $(\mu \mathrm{g})$} & \multicolumn{2}{|c|}{ Peptides identified } & \multicolumn{2}{|c|}{ Proteins quantified } \\
\hline & & & no MBR & MBR & no MBR & MBR \\
\hline \multirow{3}{*}{ FASP method } & Area 1 & 15.9 & 20074 & 31573 & 3307 & 4294 \\
\hline & Area 2 & 16.1 & 23958 & 35606 & 3658 & 4513 \\
\hline & Area 3 & 17.5 & 31941 & 41076 & 4297 & 4717 \\
\hline \multirow[t]{2}{*}{ Combined } & & & 36206 & 44048 & 4497 & 4780 \\
\hline & Area 1 & 15.9 & 19493 & 31348 & 3341 & 4327 \\
\hline \multirow[t]{2}{*}{ RapiGest method } & Area 2 & 14.6 & 23116 & 34564 & 3726 & 4513 \\
\hline & Area 3 & 16.1 & 28992 & 39075 & 4193 & 4690 \\
\hline \multirow[t]{2}{*}{ Combined } & & & 33667 & 42268 & 4424 & 4772 \\
\hline & Area 1 & 16.6 & 21653 & 32459 & 3478 & 4365 \\
\hline \multirow[t]{2}{*}{ TFE method } & Area 2 & 15.8 & 27463 & 38168 & 4051 & 4638 \\
\hline & Area 3 & 15.7 & 34430 & 42664 & 4405 & 4721 \\
\hline \multirow[t]{2}{*}{ Combined } & & & 39178 & 45224 & 4588 & 4788 \\
\hline & Area 1 & & 13100 & 23456 & 2869 & 3956 \\
\hline \multirow[t]{2}{*}{ Overlap } & Area 2 & & 15758 & 27376 & 3275 & 4280 \\
\hline & Area 3 & & 20480 & 33326 & 3842 & 4560 \\
\hline Combined & & & 48310 & 48310 & 4841 & 4841 \\
\hline
\end{tabular}

Samples were measured in 100 min single-shot DDA analysis and raw files were analyzed using MaxQuant. Recovered peptide yield ( $\mu \mathrm{g}$ ) after sample clean-up by StageTips was measured using a Nanodrop instrument. The number of peptides and proteins identified are shown with and without match-between-runs (MBR).

workflows, more than 7000 quantified protein groups have been reported from human cell lines in single $2 \mathrm{~h}$ measurements [36]. This prompted us to combine our streamlined tissue workflow with state-of-the-art DIA analysis based on the same 100 min LC gradient as in DDA analysis (see supplementary material, Supplementary materials and methods). Using high $\mathrm{pH}$ reversedphase peptide fractionation [37], we generated a project-specific spectral FFPE tissue library using our workflow, resulting in 197622 identified precursors, corresponding to 10707 protein groups (Figure 2C, D). The library covered a large number of known oncogenes and tumor suppressors with 108 members of the 135 Tumor Alterations Relevant for Genomics-driven Therapy (TARGET) set reported as 'actionable' genes [38]. By definition, alteration of these genes or their expression levels can influence clinical decisionsmaking them particularly interesting in oncology.

Across tissue samples, we consistently quantified $>5000$ protein groups in FFPE material (Figure 2E) with a low number of single peptide hits per sample (in average of 150), which led to a grouping according to tissue of origin (Figure $2 \mathrm{~F}$ ). We also included FrFr AML cells, where we quantified up to 7268 protein groups to show the applicability of our workflow to other patient material (Figure 2E). Of the 135 TARGET listed genes, almost half $(44 \%, \mathrm{~N}=59)$ were quantified on average in each sample.

We conclude that our workflow provides a robust framework for proteomic profiling of FFPE tissue samples in a 96-well format and was applicable to diverse tissue types, sample input amounts, and MS-based proteomics strategies.

\section{FFPE tissue workflow shows high quantitative reproducibility}

In addition to the robust analysis of laser microdissected samples, which showed high proteome correlations between biological tissue replicates (Figure 2B and supplementary material, Figure S2D), we assessed the reproducibility of our entire FFPE workflow for macrodissected tissues. To this end, we collected tissue areas from glass slides, as routinely used in histopathology. Of note, LCM workflows require tissue mounting on specialized glass membrane slides (e.g. polyethylene naphthalate or terephthalate (PEN or PET) that generally are not used in routine pathology. We focused on macrodissections from colorectal adenomas to reduce biological complexity within and between sections, as cancer lesions have high cellular heterogeneity at multiple levels [39]. Colorectal adenomas are benign precursor lesions to colorectal cancer. They are genetically less complex and show chromosomal stability despite their large size [40]. To assess quantitative reproducibility, we repeated the entire workflow from sample collection and processing to MS measurement and data analysis. We mounted three consecutive tissue sections obtained from four different colorectal adenomas samples, all from the same patient, on glass slides and stained them with H\&E. Based on histology, we collected the same tissue areas from all three adenoma sections using razor blade scraping. We reasoned that our biological intersection comparison between consecutive $5 \mu \mathrm{m}$ thick sections should be minor, allowing accurate estimation of the reproducibility of the entire workflow (Figure 3A, left panel). In addition, we collected three different areas from the same section to also assess intra-section proteome variability (Figure 3A, right panel). Thus, we collected 19 different tissue samples that were processed on different days. In total, 6110 protein groups were quantified in single-shot 100 min DIA analyses, with an average of 5274 per sample and a data completeness of $86 \%$. Single peptide hits comprised 345 protein groups on average, representing $6 \%$ of all quantified protein groups. To evaluate quantitative reproducibility, we calculated the coefficient of variation $(\mathrm{CV})$ for all protein groups that were quantified in at least $70 \%$ of all samples 
A

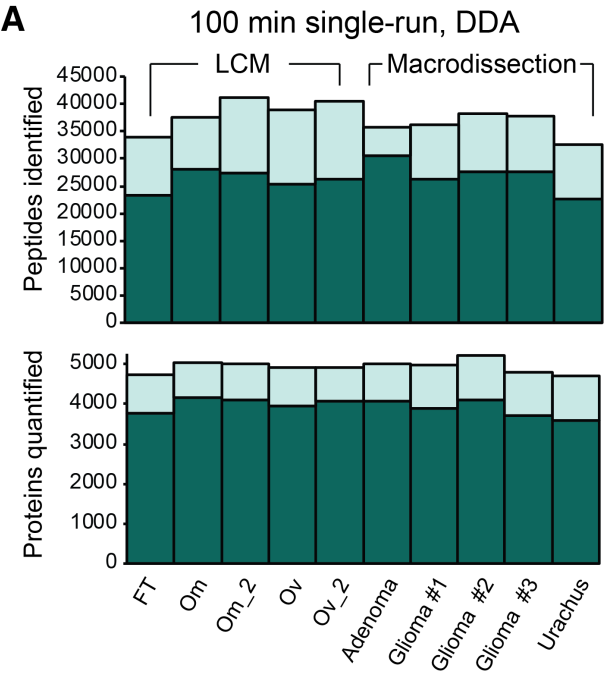

C

Library generation by high $\mathrm{pH}$
reverse-phase fractionation:
Ovarian cancer $(\mathrm{N}=6)$
Glioma $(\mathrm{N}=5)$
Adenoma $(\mathrm{N}=3)$
Acute myeloid leukemia $(\mathrm{N}=3)$

\section{D}

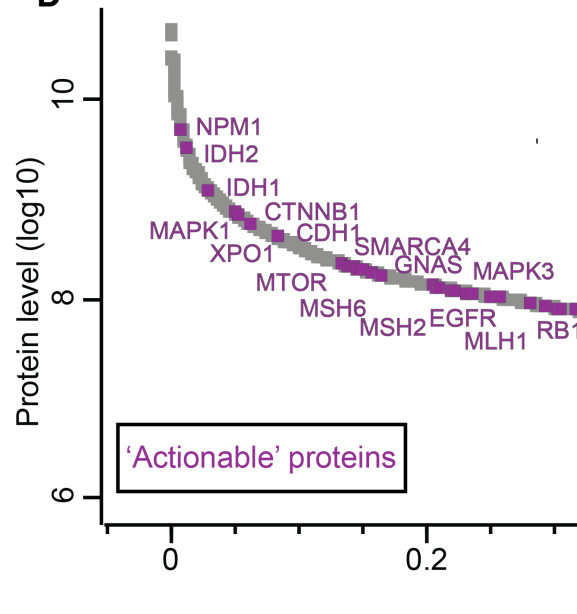

- by MS/MS

by matching

- by MS/MS

by matching
B

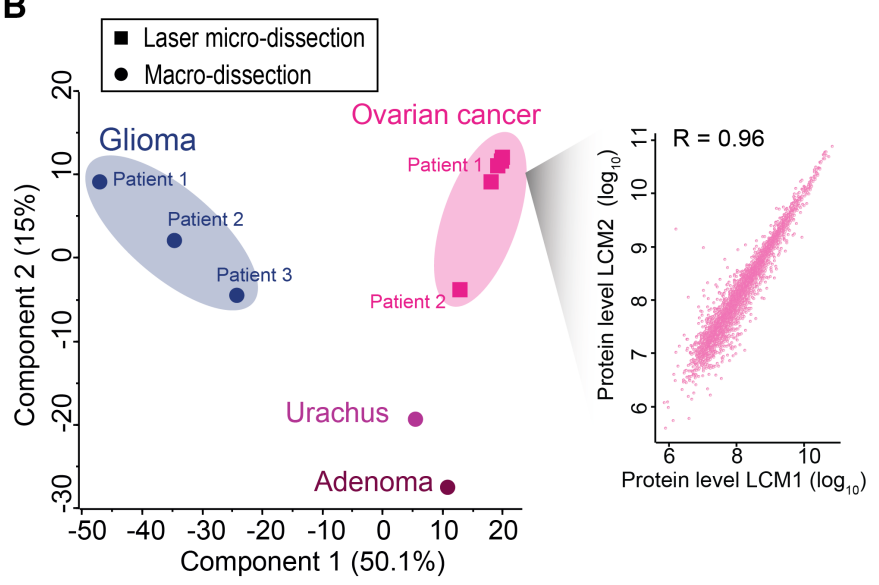

E

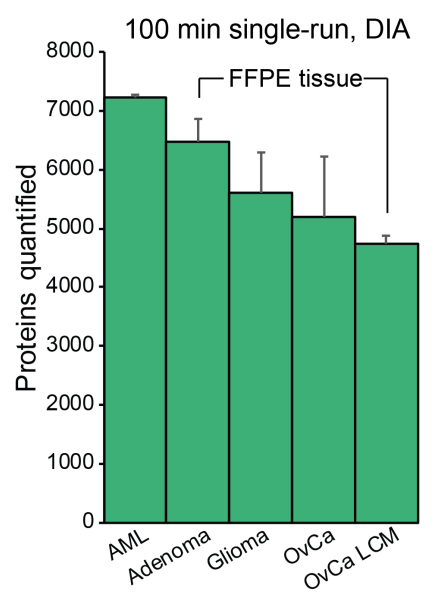

$\mathbf{F}$

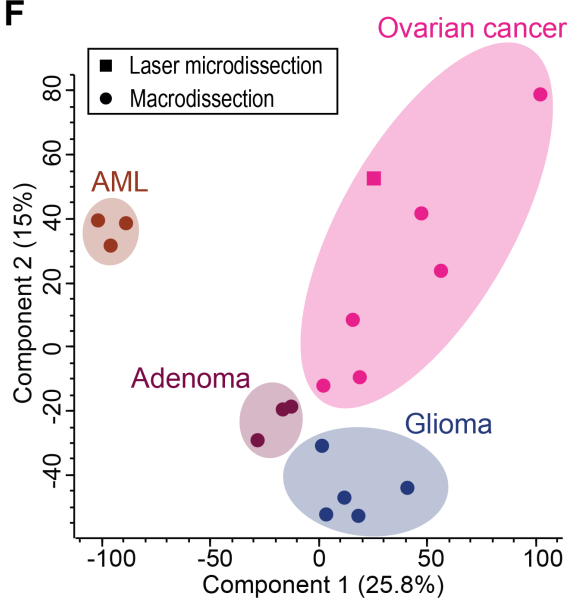

Figure 2. Evaluation of the FFPE tissue workflow in various tumor types. (A) Bar chart of the total number of identified peptides (upper panel) and quantified proteins (lower panel) by MS/MS (dark green) and matching (light green) in invasive fallopian tube (FT) cancer, omental OvCa metastasis (OM), invasive OvCa (Ov), glioma, and urachal carcinoma (urachus). The first five tissues were collected by laser-capture microdissection (LCM) and the remaining five by macrodissection. All samples were analyzed using 100 min single-shot DDA runs. (B) Principal component analysis (PCA) of the 10 tissue samples based on their proteomic expression profiles. The proteomes of glioma and OvCa samples are depicted by replicate number (patient 1-3 and collection 1-2, respectively). The first and second components segregate the samples and account for 50.1 and $15 \%$ of the variability, respectively. Ellipses encircle samples of same tissue origin. Reproducibility between two microdissected tissue samples from the same OvCa patient is depicted in the right panel (Pearson $r=0.96$ ). (C) Overview of the samples used for the generation of the spectral library by high $\mathrm{pH}$ reversed-phase fractionation. (D) Dynamic range of the MS signal of all identified proteins in the spectral library after high $\mathrm{pH}$ reversed-phase fractionation. Proteins belonging to the TARGET database, referred as actionable genes, are highlighted. (E) Average number of quantified protein groups per tissue, including acute myeloid leukemia (AML), glioma, and OvCa. Error bars show SD, minimum N = 3 replicates. (F) PCA of tissue samples measured with 100 min DIA runs. Ellipses indicate samples of same tissue origin.

(4983 protein groups). CVs were calculated across similar regions from three consecutive sections of the same FFPE block (intersection comparison) or from three different areas of the same tissue section (intrasection comparison, Figure $3 \mathrm{~A}, \mathrm{C}$ ). The intersection variability was slightly lower than intrasection variability for all analyzed adenomas (average \%CV of 18.9 and 20.1, respectively), whereas technical replicates of injections had a CV of $7.9 \%$ (Figure 3C). Pearson correlations were correspondingly high, with 0.91-0.95 for inter- and replicates and 0.97 for technical replicates (Figure 3B and supplementary 
material, Figure S3A). As about $8 \%$ of total variation originates from the LC-MS measurement alone, we estimated that sample collection and processing should contribute no more than about $10 \%$ to the total observed variation, neglecting any true biological variation. Known markers of adenoma or colorectal cancer such as E-Cadherin (CDH1), which is used clinically for diagnosis and stratification, was robustly quantified with our workflow, with CVs below or close to $20 \%$ (Figure 3D). Of note, even though EGFR was $~ 100$-fold less abundant than COX-2 (MT-CO2), its variation was somewhat lower ( $17.7 \%$ versus $24.5 \%$ ), indicating robust protein quantification even for lower abundant proteins. $\mathrm{CV}$ values across adenomas of the same patient $(\mathrm{N}=9)$ and across adenomas of different patients $(\mathrm{N}=9)$ showed higher proteome variations including known pathology markers (Figure 3E, F). Of interest, $\beta$-catenin (CTNNB1) and CDH1 showed the lowest expression differences between sections within the same and different patients, highlighting their homogeneous expression patterns in adenomas as previously reported [41,42]. Conversely, EGFR and CDX2 were variably expressed between adenomas within and between patients, again in-line with previous reports $[43,44]$. We further confirmed our proteomic results by immunohistochemistry (IHC) using validated antibodies (supplementary material, Figure S3). Low and high CDX2 or CD44 levels, quantified by proteomics, showed similar relative expression levels using IHC (Figure 3G, H) in adenoma tissues, thereby demonstrating the quality of our quantitative proteomic read-out. Taken together, these data demonstrate good workflow reproducibility and high proteome consistency within and across tissue sections of the same adenomas.

\section{Streamlined FFPE workflow applied to a cohort of 118 adenomas of different archival time}

Having developed a scalable FFPE workflow applicable to diverse sample input amounts and cancer types, we next investigated if it would allow for streamlined processing of larger tissue cohorts within a single day and with minimal hands-on pipetting time. As a proof-ofconcept, we analyzed an additional set of 118 FFPE adenoma tissues collected from 101 patients. After histopathological examination, samples were collected into 8-strip PCR tubes as before. All samples could indeed be readily processed in parallel within 1 day of sample preparation and subsequently scheduled for measurement within 10 days using the single-shot $100 \mathrm{~min}$ DIA MS method. A total of 6254 protein groups were quantified with a median of 5147 protein groups per adenoma sample (Figure 4A and supplementary material, Figure S3B). This relatively large number of adenoma tissue proteomes prompted us to investigate the molecular differences across adenomas and the impact of archival time on the proteome. The latter is of particular relevance for the design of large discovery-based studies to avoid potential sampling biases. Tissues had been collected and archived between the years 1998 and 2008 with a median archival time of 11 years and a maximum of 20 years. Samples were grouped by archival time into 14-20 years $(\mathrm{N}=14), \quad 10-13$ years $(\mathrm{N}=41)$, and 6-9 years $(\mathrm{N}=43)$. We first compared the total number of quantified peptides across archival time groups, which revealed a somewhat higher number of quantified peptides for the short-term group versus the mid-term $(-10 \%)$ and long-term groups $(-16 \%)$ (Figure 4B), independent of sample amount as reflected in the total ion current (supplementary material, Figure S4F). This trend was also apparent when we plotted the number of peptides against archival time as a continuous variable (supplementary material, Figure S4G). At the protein level, however, this trend was less pronounced, with losses of only 4 and $6 \%$, respectively. To investigate whether the lower identification rates are related to increased methionine oxidation and lysine methylation over time (the two most abundant variable peptide modifications in FFPE tissue), we included lysine methylation as a variable modification in the spectral library search and re-analyzed the DIA data. This revealed a general trend towards a higher peptide modification rate in the long-term storage samples, indicative of progressive protein modification over time [17]. However, at $1 \%$ this difference was much too small to explain the reduction of $16 \%$ in peptide identifications in the oldest samples (Figure 4B). A previous study reported lower identification rates in tissue samples stored more than 20 years, and linked it to compromised retrieval of low abundant proteins [45].

We likewise found that the peptides exclusively quantified in the short-term storage group (2719 peptides corresponding to $8 \%$ of all peptides), were of significantly lower abundance than the peptides shared across groups (Figure 4C, supplementary material, Figure S4E). A similar abundance trend was observed at the protein level but to a much lower extent $(-6 \%, 189$ protein groups unique to the short-term group) (Figure 4C, supplementary material, Figure S4E). Filtering for the 3000 most-abundant protein groups in our data set resulted in only $1 \%$ less protein quantifications in the long-term storage group (supplementary material, Figure $\mathrm{S} 4 \mathrm{H}$ ). The impact on global protein quantification was also minor, as judged by the high proteome correlations between different archival age groups (Pearson correlations 0.95-0.99) (Figure 4D). Our data are consistent with previous proteomic studies showing that protein quantification is generally not perturbed, even after 30 years of storage $[6,10,45]$. Based on these findings, we next analyzed how different archival times affected clustering into distinct proteomic subgroups. To identify the optimal number of proteomic subgroups in our data set, we applied a consensus clustering approach [24] based on the 1000 most variably expressed protein groups across adenomas as measured by median absolute deviation. Three samples were excluded from the analysis due to low protein identifications. This resulted in four major proteome clusters of similar size, differing in their protein expression profiles (Figure $4 \mathrm{E}, \mathrm{G}$, and supplementary material, Figure S4C, D). We observed 
A

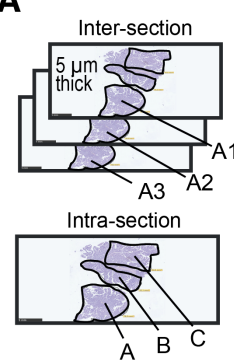

D
B

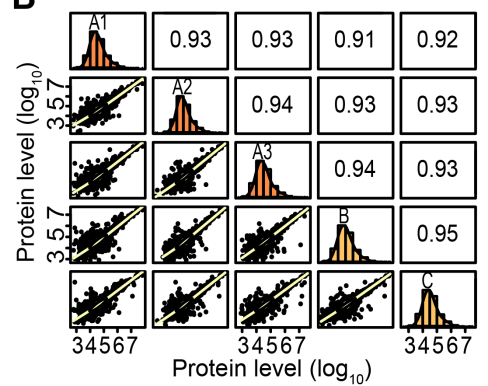

C

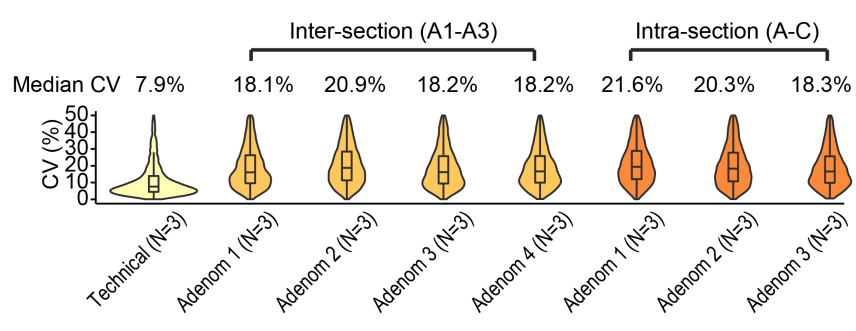

E
$\mathbf{F}$

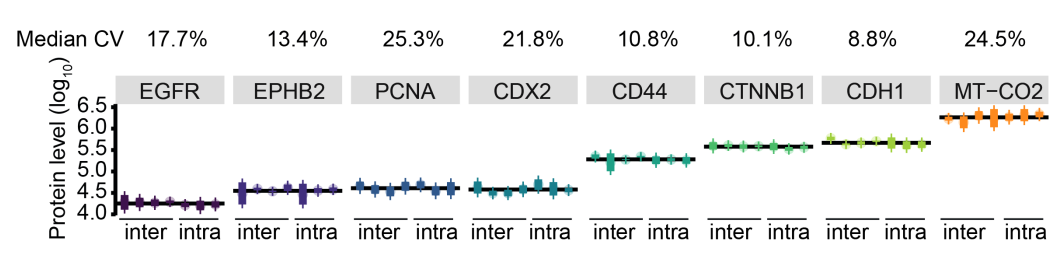

G
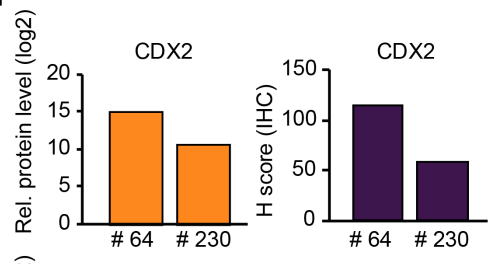

H
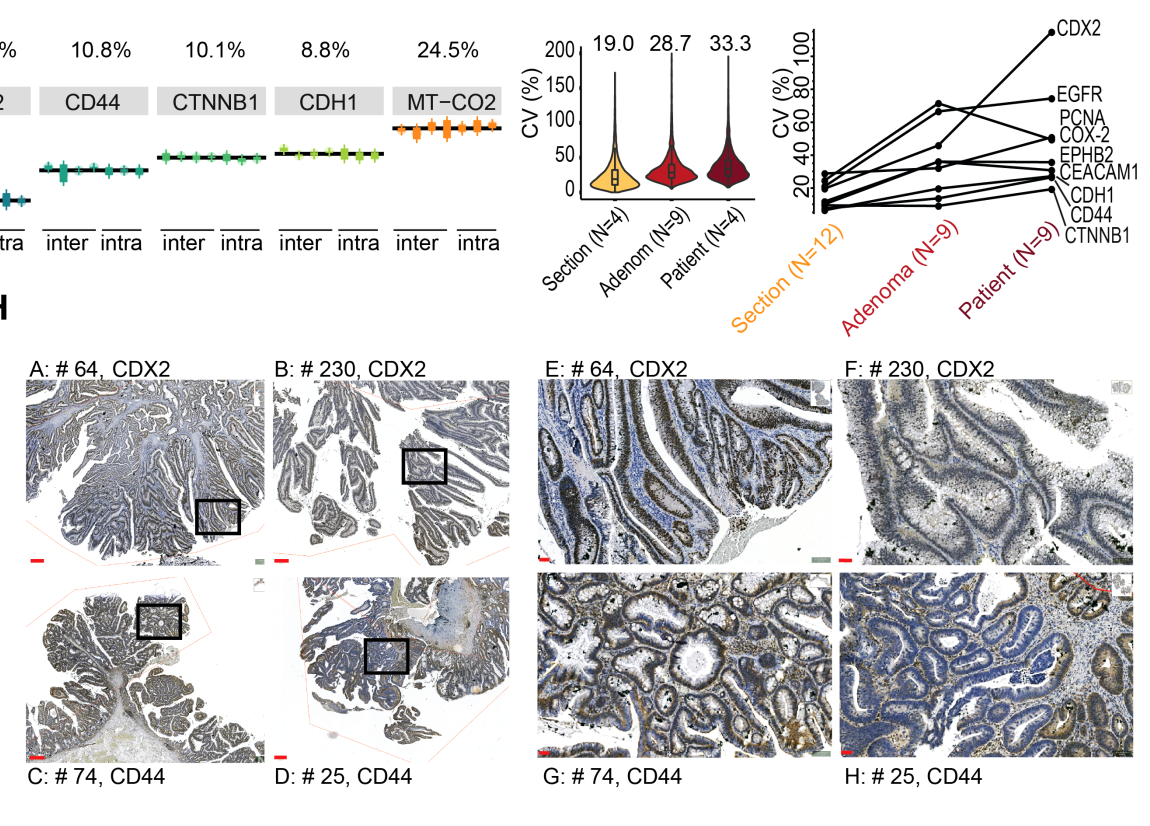

G: \# 74, CD44

$\mathrm{H}:$ \# 25, CD44

Figure 3. Evaluation of the reproducibility of the FFPE tissue workflow. (A) Schematic representation of the H\&E-stained adenoma tissue sections used for overall workflow reproducibility assessment. Inter- and intrasection proteomic variability assessment is depicted in the upper and lower panels, respectively. Circles indicate the tissue regions manually collected by macrodissection. (B) Proteome correlation matrix of inter- $(\mathrm{A} 1-\mathrm{A} 3)$ and intrasection $(\mathrm{A}-\mathrm{C})$ replicates of the same FFPE adenoma tissue. Depicted values are Pearson correlations. (C) Box plots and violin plots show the coefficient of variations (CVs in \%) of protein quantification across injection replicates $(\mathrm{N}=3)$, intersection replicates (four sections of three replicates each), and intrasection replicates (three sections of three replicates each) based on 4983 protein groups. For simplicity, values above 50\% were excluded from the plots. CVs were calculated based on non-logarithmic values. (D) Median CV values are plotted for eight known adenoma and colorectal cancer (CRC) markers across inter- (four values) and intra- (three values) section replicates. The average abundance for each protein across all sections is shown as a black line. Median CV values (\%) for each protein across all sections are displayed above the plot. CVs were calculated based on non-logarithmic values. (E) CV values of protein quantification across adenoma sections $(N=4$, same adenoma), adenoma tissues $(N=9$, same patient), and patients $(N=9$, different patients). (F) CV values of nine quantified adenoma and CRC marker proteins across different sections, tissues, and patients. (G) Comparison between relative protein quantification based on proteomics (orange) and IHC (H-score, purple) for CDX2 and CD44 in four adenoma patients. (H) IHC staining of $C D X 2$ or $C D 44$ for four adenoma tissues. Black boxes show representative regions that are enlarged in the right panels $(E-H)$. Scale bars, $400 \mu \mathrm{m}(\mathrm{A}, \mathrm{B}, \mathrm{D}), 500 \mu \mathrm{m}(\mathrm{C})$, and $50 \mu \mathrm{m}(\mathrm{E}-\mathrm{H})$.

some overlap across the four clusters, indicative of shared biological features (Figure 4G). For instance, proteins related to mitochondria and cellular respiration were significantly higher in clusters 1,3 , and 4 versus cluster 2, whereas cell adhesion and immune system related proteins were higher in clusters 2,3 , and 4 versus cluster 1 . Reassuringly, biological variability between samples was more dominant than variability caused by archival time, and as a result archival groups were subdivided into the four separate clusters (Figure 4F, G). This further illustrates the general applicability of FFPE tissues for proteomics-centered disease phenotyping and patient stratification.

\section{Discussion}

FFPE blocks are the most commonly used format for storing tissues in pathology due to its long-storage ability at room temperature, simplicity, and low-cost. Hundreds of millions of FFPE samples exist worldwide in tissue biobanks, potentially available for analysis. These immense archives represent an invaluable resource and opportunity to study molecular mechanisms of the diverse diseases for which tissue samples are taken. MS-based proteomics has been used increasingly over the last years to investigate FFPE samples at the proteome level, but this typically required tedious workflows, 
A
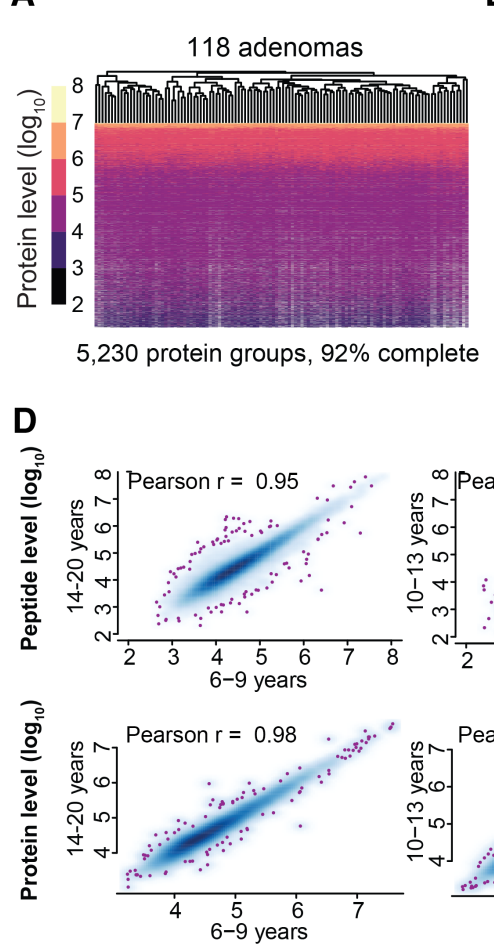

$\mathbf{F}$

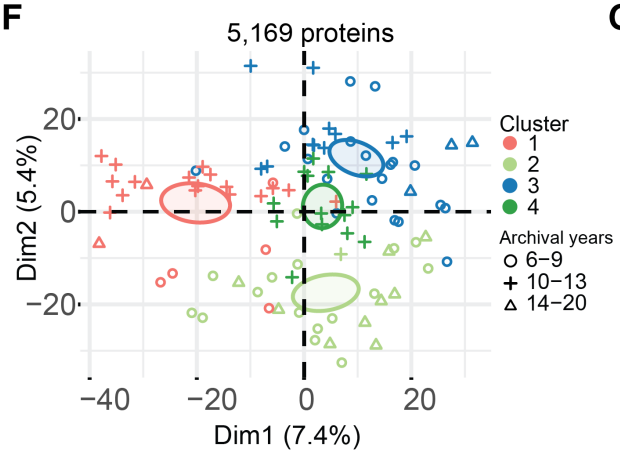

B

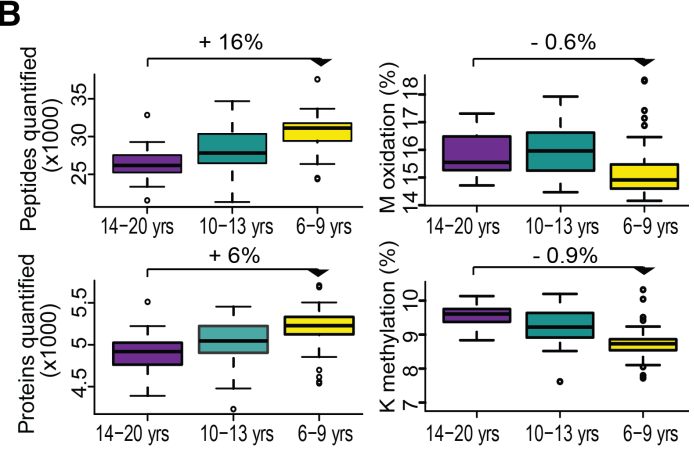

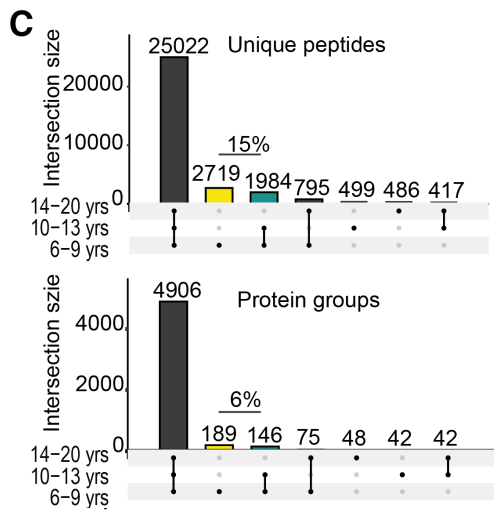

E

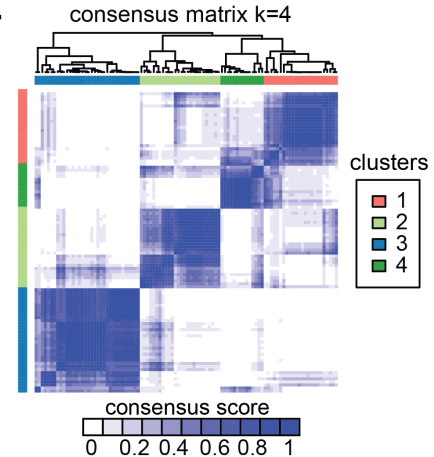

G

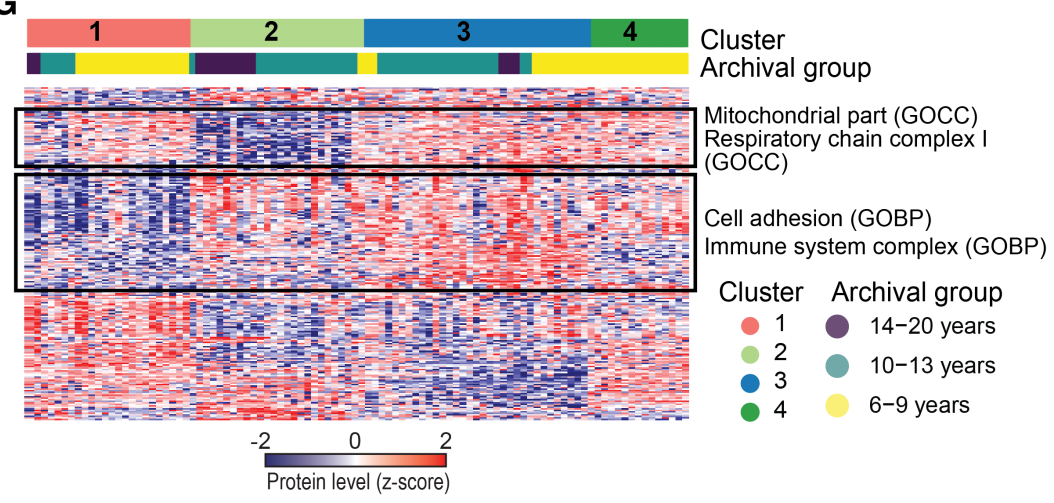

Figure 4. Streamlined proteomic analysis of 118 adenoma tissues of varying archival time. (A) Heat map of protein abundance across 118 adenoma tissues encompassing 5230 protein groups. Missing values are shown in white. Unsupervised hierarchical clustering was performed column-wise with Pearson correlations as distance metric. (B) Box plots displaying the total number of quantified peptides and proteins, as well as lysine methylation and methionine oxidation in FFPE tissues archived with storage time ranging from 6 to 20 years, grouped into three intervals: $14-20$ years $(N=14), 10-13$ years $(N=41)$, and 6-9 years $(N=43)$. For each group, a minimum of $50 \%$ quantified values was required. (C) UpSet plots showing intersections of peptide (upper panel) and protein (lower panel) quantification across archival time groups. (D) Scatterplot of pairwise proteomic comparisons between archival groups on peptide (upper panels) and protein level (lower panels). (E) Consensus clustering of the proteomic expression data determined by Pearson correlation as distance metric, where $k$ represents the total number of clusters. Consensus scores are indicated using a color scale from white (samples never cluster together) to blue (samples always cluster together). (F) PCA of 101 colorectal adenoma samples based on their proteomic expression profiles, encompassing 5169 proteins. The first and second dimensions segregate the samples and account for 7.4 and $5.4 \%$ of the total variability, respectively. The color code corresponds to the four adenoma subclusters identified in $(E)$ whereas symbol types indicate the three archival groups. Point concentration ellipses are shown for each cluster with a $95 \%$ confidence interval. (G) Co-regulated pathways across the four adenoma clusters. Heat map of $z$-scored protein abundances of the 416 differentially expressed proteins (ANOVA, FDR $<0.01, s_{0}=0.5$ ) after unsupervised hierarchical clustering. Up- and downregulated proteins are represented in red and blue, respectively. Significantly enriched pathways (Benjamini-Hochberg FDR $<0.05$, GOCC, and GPBP terms) are shown exemplarily for co-expressed protein clusters across the four patient groups. Archival and cluster groups are indicated as color bar.

and lacked reproducibility, throughput, and analytical depth. This prompted us to develop a streamlined and robust MS-based workflow for the proteomic investigation of many FFPE samples.

With our new workflow in hand, we demonstrated the practicality of analyzing relatively large clinical FFPE cohorts from pathology glass slides with good proteomic depth in single-run analysis using state-of-the-art DDA and DIA acquisition methods. Based on a single-tube workflow in 96-well format and using MS compatible protein extraction buffers, we demonstrated high proteome similarity to detergent-based sample preparation 
and achieve a proteomic depth of 5000-6500 protein groups per single sample and in diverse tissue types. As tissue amounts are often highly variable in histopathology, it was critical to make our workflow applicable also to low sample amounts. To this end, we showed that laser-microdissected samples, encompassing less than 10000 cells, can be analyzed in a reproducible fashion. We also demonstrated that FFPE tumor types of different entities can be reproducibly processed with our workflow as required in pathology practice. We retrieved known biological differences between $\mathrm{OvCa}$, glioma, and urachal carcinoma, validating the overall quality of our workflow. Using unbiased MS-based proteomics, we investigated the cancer proteomes on a large scale and showed their potential to detect and quantify a large proportion of known 'actionable' proteins. In contrast to antibody-based methods that require prior knowledge of the proteins of interest, we here investigate thousands of additional proteins for a better understanding of the underlying disease mechanisms. We envision that the proteomic analysis of FFPE tissues will be clinically relevant in the future, in particular when sequence and splice variants are also considered. Our short sample preparation time of less than 1 day, followed by prompt MS measurement and data analysis, highlights the promise of our FFPE workflow in future clinical pathology practice, where fast sample analysis for diagnosis and target identification in patients is key. It is pertinent that our workflow can be well integrated with routine tissue preparation in pathology, where direct tissue collection from glass slides of H\&E-stained sections offers several important advantages. Separate de-paraffinization, which usually comes at the cost of throughput and sample preparation time, is not needed, as it is already part of standardized H\&E-staining procedures. Furthermore, the availability of image data, collected routinely in histopathology for patient diagnosis, can be integrated to guide sample collection and to avoid contamination from nondiseased tissue areas, which is often a major limitation for bulk tissue analysis. This is illustrated in our analysis of adenoma tissues where we observed a high degree of proteomic similarity between overlapping tissue areas across sections of the same FFPE block. In this tissue type, we further investigated the impact of archival time on several proteome quality parameters including peptide and protein quantifications, as well as peptide modifications. Although the literature reports conflicting results on the impact of archival time on the number of identified peptides and protein groups depending on different archival times, we noticed a drop of $16 \%$ in peptide identifications in long-term storage samples (14-20 years), compared to 6- to 9-year-old samples. However, one 15-year-old sample showed a similar number of peptide identifications compared to the average of the 6- to 9-year-old samples (supplementary material, Figure $\mathrm{S4G}$ ), suggesting that good tissue processing and storage could alleviate this effect $[17,46]$. In any case, we observed a 'buffer' effect on the protein level as the decrease in peptide quantification translated only to a drop of $6 \%$ at the protein level, which sustains global proteome integrity of old samples. This highlights the advantage of shotgun proteomic approaches that typically integrate information from multiple peptides for protein quantification. Our data further demonstrate that the quantitative protein information was not perturbed by storage time as we observed high global proteome correlations between storage groups. This suggests that previously published conflicting results might have arisen from differences in proteome coverage due to the workflows employed. Filtering our data set for the most abundant proteins revealed a drop of only $1 \%$ of protein quantifications between the different storage groups. Although additional investigation will be required to fully address the impact of archival time on the proteome, we found that biological differences across adenoma tissues drive the segregation of samples independently of archival times. Our analysis revealed previously unknown adenoma clusters, reflecting potential new subtypes that could lead to a novel classification. This speculation, however, will require a more thorough molecular analysis to validate these preliminary data.

In the future, our proteomic FFPE workflow could be extended to phospho-proteomics that will require streamlined analyses at the PTM level, as well as even greater sensitivity. Information at the phosphorylation level is often crucial to understand disease biology, in particular to reflect kinase activity driving tumor development. Furthermore, combined with genomic information, multi-omics data could further improve current molecular diagnosis [47]. This combination would confirm whether a reported kinase mutation leads to kinase activation or inactivation to better understand its role in cancer development and progression.

To this end, promising developments of highthroughput enrichment methods, using robotic assistance, have been developed and are becoming more practical. For instance, automated phospho-peptide enrichment can be achieved in only $1 \mathrm{~h}$ [48], representing a promising avenue for FFPE tissues analysis. Finally, single-cell technologies are becoming increasingly important to profile genetic, epigenetic, spatial, proteomic, and lineage information of individual cells [49]. We believe that a variation of such single-cell approaches could also be developed for FFPE analyses. For all these reasons, streamlined, robust, and scalable workflows for FFPE tissue analysis, such as the one introduced here, will be of immense value.

In conclusion, we demonstrated a high-throughput and reproducible proteomic workflow that now enables researchers to analyze large clinical FFPE tissue cohorts with wide-ranging sample amounts in a robust, timely, and streamlined manner. We hope that will help triggering the proteomic analysis of thousands of valuable FFPE tissues. This information can then be combined with other 'omic' data, with the ultimate goal of uncovering tissue biomarkers for better patient classification, diagnosis, or treatment. 


\section{Acknowledgements}

We thank all members of the department of Proteomics and Signal Transduction at the Max Planck Institute of Biochemistry, and the Clinical Proteomics Group at the The Novo Nordisk Foundation Center for Protein Research, for support and fruitful discussions. In particular, we thank Jakob Bader and Arend Koch (Charité Berlin) for assistance with glioma tissue analysis, Philipp Nuhn, Maximilian C. Kriegmair, and Stefan Porubsky (Mannheim medical center) for providing urachal FFPE samples; Annette Bartels for assistance with colorectal adenoma tissue collection and preparation; Ashok Kumar Jayavelu for assistance with AML collection and analysis; and Philipp Geyer for sample preparation input. This work was supported by the Max Planck Society for the Advancement of Science and by the Novo Nordisk Foundation (grant agreement NNF14CC0001 and NNF15CC0001), the European Union's Horizon 2020 research and innovation programme under grant agreement 686547 (MSmed Project) and 846795 (Marie Skłodowska-Curie grant), and the University of Chicago Cancer Center Support Grant P30CA014599.

An unreviewed version of this manuscript was placed on bioRxiv (https://doi.org/10.1101/779009) on September 23, 2019.

\section{Author contributions statement}

FC, SD, and MM conceived the study. FC and SD performed proteomic sample preparation, analysis, and interpretation under supervision by MM. FC and SD designed experiments. EL collected and selected OvCa tissues and FC microdissected them. JL and GIM collected and selected adenoma tissues for the study. JB prepared adenomas for proteomics, and FC and AM processed them. FC, SD, and LS prepared Figures. FC, SD, and MM wrote the manuscript and FC, SD, JB, AM, EL, JM, LS, and MM edited it. All authors reviewed and provided feedback on the manuscript.

\section{Data availability statement}

The mass spectrometry proteomics data have been deposited to the ProteomeXchange Consortium (http:// proteomecentral.proteomexchange.org/cgi/GetDataset) via the PRIDE partner repository with the data set identifier PXD017269 without restriction. All other data supporting the findings of this study are available within the article and its supplementary information files or from the corresponding author upon reasonable request.

\section{References}

1. Grillo F, Bruzzone M, Pigozzi S, et al. Immunohistochemistry on old archival paraffin blocks: is there an expiry date? J Clin Pathol 2017; 70: 988-993.
2. Wu W, Choudhry H (eds). Next Generation Sequencing in Cancer Research. Springer: New York, 2013.

3. Arreaza G, Qiu P, Pang L, et al. Pre-analytical considerations for successful next-generation sequencing (NGS): challenges and opportunities for formalin-fixed and paraffin-embedded tumor tissue (FFPE) samples. Int J Mol Sci 2016; 17.

4. Strom SP. Fundamentals of RNA analysis on biobanked specimens. Methods Mol Biol 1897; 2019: 345-357.

5. Scalia CR, Boi G, Bolognesi MM, et al. Antigen masking during fixation and embedding, dissected. J Histochem Cytochem 2017; 65: 5-20.

6. Ostasiewicz P, Zielinska DF, Mann M, et al. Proteome, Phosphoproteome, and N-Glycoproteome are quantitatively preserved in formalin-fixed paraffin-embedded tissue and analyzable by highresolution mass spectrometry. J Proteome Res 2010; 9: 3688-3700.

7. Wakabayashi M, Yoshihara H, Masuda T, et al. Phosphoproteome analysis of formalin-fixed and paraffin-embedded tissue sections mounted on microscope slides. J Proteome Res 2014; 13: 915-924.

8. Wisniewski JR, Dus K, Mann M. Proteomic workflow for analysis of archival formalin-fixed and paraffin-embedded clinical samples to a depth of 10000 proteins. Proteomics Clin Appl 2013; 7: 225-233.

9. Kennedy JJ, Whiteaker JR, Schoenherr RM, et al. Optimized protocol for quantitative multiple reaction monitoring based proteomic analysis of formalin-fixed, paraffin-embedded tissues. $J$ Proteome Res 2016; 15: 2717-2728.

10. Piehowski PD, Petyuk VA, Sontag RL, et al. Residual tissue repositories as a resource for population-based cancer proteomic studies. Clin Proteomics 2018; 15: 26.

11. Aebersold R, Mann M. Mass-spectrometric exploration of proteome structure and function. Nature 2016; 537: 347-355.

12. Coscia F, Lengyel E, Duraiswamy J, et al. Multi-level proteomics identifies CT45 as a Chemosensitivity mediator and immunotherapy target in ovarian cancer. Cell 2018; 175: 159-170 e116.

13. Mertins P, Mani DR, Ruggles KV, et al. Proteogenomics connects somatic mutations to signalling in breast cancer. Nature 2016; 534: $55-62$.

14. Zhang H, Liu T, Zhang Z, et al. Integrated proteogenomic characterization of human high-grade serous ovarian cancer. Cell 2016; 166: 755-765.

15. Magdeldin S, Yamamoto T. Toward deciphering proteomes of formalin-fixed paraffin-embedded (FFPE) tissues. Proteomics 2012; 12: 1045-1058.

16. Wang HX, Qian WJ, Mottaz HM, et al. Development and evaluation of a micro- and nanoscale proteomic sample preparation method. J Proteome Res 2005; 4: 2397-2403.

17. Sprung RW, Brock JWC, Tanksley JP, et al. Equivalence of protein inventories obtained from formalin-fixed paraffin-embedded and frozen tissue in multidimensional liquid chromatography-tandem mass spectrometry shotgun proteomic analysis. Mol Cell Proteomics 2009; 8: 1988-1998.

18. Yu YQ, Gilar M, Lee PJ, et al. Enzyme-friendly, mass spectrometrycompatible surfactant for in-solution enzymatic digestion of proteins. Anal Chem 2003; 75: 6023-6028.

19. Kulak NA, Pichler G, Paron I, et al. Minimal, encapsulated proteomic-sample processing applied to copy-number estimation in eukaryotic cells. Nat Methods 2014; 11: 319-U300.

20. Foll MC, Fahrner M, Oria VO, et al. Reproducible proteomics sample preparation for single FFPE tissue slices using acid-labile surfactant and direct trypsinization. Clin Proteomics 2018; 15: 11.

21. Kelstrup CD, Bekker-Jensen DB, Arrey TN, et al. Performance evaluation of the Q Exactive HF-X for shotgun proteomics. J Proteome Res 2018; 17: 727-738.

22. Cox J, Mann M. MaxQuant enables high peptide identification rates, individualized p.p.b.-range mass accuracies and proteome-wide protein quantification. Nat Biotechnol 2008; 26: 1367-1372. 
23. Tyanova S, Temu T, Sinitcyn P, et al. The Perseus computational platform for comprehensive analysis of (prote)omics data. Nat Methods 2016; 13: 731-740.

24. Wilkerson MD, Hayes DN. ConsensusClusterPlus: a class discovery tool with confidence assessments and item tracking. Bioinformatics 2010; 26: 1572-1573.

25. Thorsen SF, Gromova I, Christensen IJ, et al. Gel-based proteomics of clinical samples identifies potential serological biomarkers for early detection of colorectal cancer. Int J Mol Sci 2019; 20: pii: E6082.

26. Eckert MA, Coscia F, Chryplewicz A, et al. Proteomics reveals NNMT as a master metabolic regulator of cancer-associated fibroblasts. Nature 2019; 569: 723-728.

27. Wisniewski JR, Zougman A, Nagaraj N, et al. Universal sample preparation method for proteome analysis. Nat Methods 2009; 6: 359-362.

28. Davis S, Scott C, Ansorge O, et al. Development of a sensitive, scalable method for spatial, cell-type-resolved proteomics of the human brain. J Proteome Res 2019; 18: 1787-1795.

29. Cox J, Hein MY, Luber CA, et al. Accurate proteome-wide label-free quantification by delayed normalization and maximal peptide ratio extraction, termed MaxLFQ. Mol Cell Proteomics 2014; 13: 2513-2526.

30. Puchtler H, Meloan SN. On the chemistry of formaldehyde fixation and its effects on Immunohistochemical reactions. Histochemistry 1985; 82: 201-204.

31. Metz B, Kersten GFA, Hoogerhout P, et al. Identification of formaldehyde-induced modifications in proteins - reactions with model peptides. J Biol Chem 2004; 279: 6235-6243.

32. Chi H, Liu C, Yang H, et al. Comprehensive identification of peptides in tandem mass spectra using an efficient open search engine. Nat Biotechnol 2018; 36: 1059.

33. Zhang Y, Muller M, Xu B, et al. Unrestricted modification search reveals lysine methylation as major modification induced by tissue formalin fixation and paraffin embedding. Proteomics 2015; 15: 2568-2579.

34. Kawashima Y, Kodera Y, Singh A, et al. Efficient extraction of proteins from formalin-fixed paraffin-embedded tissues requires higher concentration of tris(hydroxymethyl)aminomethane. Clin Proteomics 2014; 11: 4 .

35. Meier F, Geyer PE, Virreira Winter S, et al. BoxCar acquisition method enables single-shot proteomics at a depth of 10,000 proteins in 100 minutes. Nat Methods 2018; 15: 440-448.

36. Bruderer R, Bernhardt OM, Gandhi T, et al. Optimization of experimental parameters in data-independent mass spectrometry significantly increases depth and reproducibility of results. Mol Cell Proteomics 2017; 16: 2296-2309.

37. Kulak NA, Geyer PE, Mann M. Loss-less Nano-fractionator for high sensitivity, high coverage proteomics. Mol Cell Proteomics 2017; 16: 694-705.

38. Van Allen EM, Wagle N, Stojanov P, Perrin DL, Cibulskis K, Marlow S, Jane-Valbuena J, Friedrich DC, Kryukov G, Carter SL,
McKenna A, Sivachenko A, Rosenberg M, et al. Whole-exome sequencing and clinical interpretation of formalin-fixed, paraffinembedded tumor samples to guide precision cancer medicine. Nat Med 2014; 20: 682-688.

39. Gay L, Baker AM, Graham TA. Tumour cell heterogeneity. F1000Res 2016: 5: F1000 Faculty Rev-238.

40. Sottoriva A, Kang H, Ma Z, et al. A big bang model of human colorectal tumor growth. Nat Genet 2015; 47: 209-216.

41. Pap Z, Pavai Z, Denes L, et al. An Immunohistochemical study of colon adenomas and carcinomas: E-cadherin, Syndecan-1, Ets-1. Pathol Oncol Res 2009; 15: 579-587.

42. Iwamoto M, Ahnen DJ, Franklin WA, et al. Expression of betacatenin and full-length APC protein in normal and neoplastic colonic tissues. Carcinogenesis 2000; 21: 1935-1940.

43. Williet N, Petcu CA, Rinaldi L, et al. The level of epidermal growth factor receptors expression is correlated with the advancement of colorectal adenoma: validation of a surface biomarker. Oncotarget 2017; 8: 16507-16517.

44. Bakaris S, Cetinkaya A, Ezberci F, et al. Expression of homeodomain protein CDX2 in colorectal adenoma and adenocarcinoma. Histol Histopathol 2008; 23: 1043-1047.

45. Balgley BM, Guo T, Zhao K, et al. Evaluation of archival time on shotgun proteomics of formalin-fixed and paraffin-embedded tissues. J Proteome Res 2009; 8: 917-925.

46. Thompson SM, Craven RA, Nirmalan NJ, et al. Impact of preanalytical factors on the proteomic analysis of formalin-fixed paraffin-embedded tissue. Proteom Clin Appl 2013; 7: 241-251.

47. Doll S, Gnad F, Mann M. The case for proteomics and Phosphoproteomics in personalized cancer medicine. Proteom Clin Appl 2019; 13: e1800113.

48. Post H, Penning R, Fitzpatrick MA, et al. Robust, sensitive, and automated Phosphopeptide enrichment optimized for low sample amounts applied to primary hippocampal neurons. J Proteome Res 2017; 16: 728-737.

49. Stuart T, Satija R. Integrative single-cell analysis. Nat Rev Genet 2019; 20: 257-272.

*50. Coscia F, Watters KM, Curtis M, et al. Integrative proteomic profiling of ovarian cancer cell lines reveals precursor cell associated proteins and functional status. Nat Commun 2016; 7: 12645.

*51. Rappsilber J, Mann M, Ishihama Y. Protocol for micro-purification, enrichment, pre-fractionation and storage of peptides for proteomics using StageTips. Nat Protoc 2007; 2: 1896-1906.

*52. Wichmann C, Meier F, Winter SV, et al. MaxQuant. Live enables global targeting of more than 25,000 peptides. Mol Cell Proteomics 2019; 18: 982-994.

*53. Cox J, Neuhauser N, Michalski A, et al. Andromeda: a peptide search engine integrated into the MaxQuant environment. J Proteome Res 2011; 10: 1794-1805.

*Cited only in supplementary material.

\section{SUPPLEMENTARY MATERIAL ONLINE \\ Supplementary materials and methods \\ Supplementary figure legends}

Figure S1. Mass spectrometry-based proteomic workflow for FFPE tissues

Figure S2. FFPE tissue workflow is broadly applicable across tissue types

Figure S3. Immunohistochemical control stains

Figure S4. Reproducible and streamlined proteomic analysis of adenoma tissues of varying archival time 